JGG 2021;69:130-132

doi: $10.36150 / 2499-6564-N 340$

\title{
A cascade of complications in a hospitalized frail older patient: is a better management possible?
}

Ambra Bertola, Sarah Damanti, Raffaella Scotti, Giuseppe Di Lucca, Enrica Bozzolo, Moreno Tresoldi

Unit of General Medicine and Advanced Care, IRCCS San Raffaele Hospital, Milan, Italy

Methods. Describing the complications that an older patient underwent during his hospital stay.

Results. An 87-year-old man was admitted to the San Raffaele Hospital for a transient loss of consciousness (LOC). He was hospitalized for assessing the causes of the LOC. The LOC lasted 2 minutes, was associated with bilateral upper limb clonus and subsequent confusion. The patient was severely comorbid: he suffered from metabolic syndrome, chronic kidney failure, hemodynamically significant carotid atheromasia, anemia, and he had a dubious history of epilepsy. Moreover, the patient was cognitively impaired and many episodes of LOC were reported in his medical history. During the hospital stay the patient developed many complications: a fall, nosocomial pneumonia, urinary retention and deconditioning.

Received: December 24, 2020

Accepted: February 15, 2021

\section{Correspondence \\ Sarah Damanti \\ Unit of General Medicine and Advanced Care, IRCCS San Raffaele Hospital, via Olgettina 60, Milan, Italy \\ E-mail: sarah.damanti@hotmail.it}

\section{Conflict of interest}

The Authors declare no conflict of interest

How to cite this article: Bertola $A$, Damanti S, Scotti R, et al. A cascade of complications in a hospitalized frail older patient: is a better management possible? Journal of Gerontology and Geriatrics 2021;69:130-132. https://doi. org/10.36150/2499-6564-N340

C Copyright by Società Italiana di Gerontologia e Geriatria (SIGG)

\section{(c) (i) $(9)$}

\section{OPEN ACCESS}

This is an open access article distributed in accordance with the CC-BY-NC-ND (Creative Commons Attribution-NonCommercial-NoDerivatives 4.0 International) license. The article can be used by giving appropriate credit and mentioning the license, but only for non-commercial purposes and only in the original version. For further information: https://creativecommons.org/licenses/by-nc-nd/4.0/deed.en
Conclusions. Global aging poses several challenges to society and public health systems. The traditional models of care are not suitable to face the complex needs of frail older people for whom standard decisional algorithms are seldom applicable. The context of hospitalization, though necessary, may be hazardous risky for the health of older people, especially the frailer ones.

Key words: frailty, hospitalization, complications

\section{INTRODUCTION}

Global aging poses several challenges to society and public health systems. The traditional models of care are not suitable to face the complex needs of frail older people for whom standard decisional algorithms are seldom applicable. The context of hospitalization, though necessary, may be hazardous risky for the health of older people, especially the frailer ones. We describe the case of a frail older patient who underwent several complications while he was hospitalized for a loss of consciousness.

\section{CASE DESCRIPTION}

An 87-year-old man was admitted to the San Raffaele Hospital for a transient loss of consciousness (LOC), which occurred in the morning after defecation. The LOC lasted 2 minutes, was associated with bilateral upper limb clonus and subsequent confusion. The patient was severely comorbid: 
he suffered from metabolic syndrome, chronic kidney failure, hemodynamically significant carotid atheromasia (with a complete occlusion of the left internal carotid artery and a previous thromboendarterectomy at the level of the right internal carotid artery), anemia, and he had a dubious history of epilepsy (in the absence of pharmacological treatment). Moreover, the patient was cognitively impaired and many episodes of LOC were reported in his medical history. For another LOC he recently underwent cardiovascular investigations (echocardiogram and echocolordoppler of the supraortic trunks), which excluded cardiac causes of the LOC. On presentation to the emergency department his physical examination was normal except for hypoxemia at pulsioxymeter ( $90 \%$ in room air). Blood tests showed an increased $\mathrm{C}$ reactive protein $(97 \mathrm{mg} / \mathrm{l})$, and a worsening of the renal function. Glycaemia was normal. The chest $X$ ray and the urine exam were normal. Considering the history of epilepsy, though doubtful, the patient underwent an electroencephalogram (EEG) which showed an asymmetric alteration of the general organization.

During the Emergency Department (ED) stay the patient fell off the stretcher. Therefore a head CT scan was done, that was negative for hemorrhagic alterations.

The patient was treated with ceftriaxone and metronidazole, oxygen supply and urinary catheter placement. In addition, the neurologist consultant suggested an antiepileptic therapy with levetiracetam. Then he was admitted to the General Medicine Unit of the Hospital.

To investigate the causes of the LOC, a cardiac Holter was attempted. Unfortunately, confusion and agitation due to his cognitive impairment caused him to remove it after few hours. It was also impossible to perform the tests for orthostatic hypotension because the patient was unable to stand upright, even if supported. A neurologic re-evaluation concluded for the diagnosis of convulsive syncope. Therefore, levetiracetam was withdrawn.

The hospital stay was complicated by nosocomial pneumonia, for whom the patient received further antibiotic therapy with piperacilline/tazobactam, and by acute urinary retention after an attempt to remove the bladder catheter. Last, but not least, as a consequence of the prolonged bed rest the patient developed serious deconditioning, which further impaired his ability to change posture, stand and walk (Fig. 1).

\section{DISCUSSION}

Comorbidities and social issues (i.e. psychological and socio-economic problems) can reduce the reserve capacity of older people, making them more vulnerable. This condition is named frailty ${ }^{1}$. Frail people are at

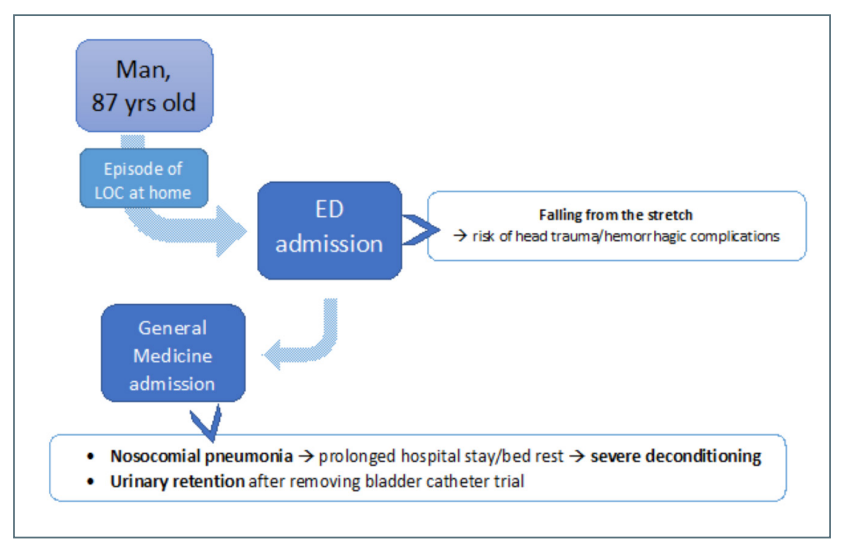

Figure 1. Real management of the patient.

increased risk of developing adverse clinical outcomes when exposed to stressors like a disease. For example they can develop disability, be hospitalized or institutionalized or can die ${ }^{2,3}$

Frailty affects millions of people worldwide, and the number of frail people is rapidly increasing. Besides having an impact on the quality of life of the affected people frailty challenges the long-term sustainability of health care systems.

The traditional models of care are not suitable to face the complex needs of frail older people for whom standard decisional algorithms are seldom applicable. The context of hospitalization, though necessary, may be hazardous risky for the health of older people, especially the frailer ones. Indeed, our patient developed many complications during his hospital stay: a fall, nosocomial pneumonia, urinary retention and deconditioning.

A geriatric approach to this patient should have been started in the ED. Unfortunately the overcrowding of the ED associated with the limited geriatric culture of the emergency physicians and the lack of adequate territorial network of services for the management of older patients outside the hospital lead to the inappropriate hospitalization of this patient. During the hospital stay we started a geriatric approach by de-prescribing the inappropriate antiepileptic medications. However a vicious circle had already started producing a series of complications that prolonged the hospital stay.

Cognitive impairment, disability, and comorbidities were demonstrated to predict hospitalization in older people ${ }^{3,4}$. Thus, the frailest individuals, for whom hospitalization can be a harmful event, are those at highest risk to be hospitalized. Hospitalizing frail individuals can dramatically increase the healthcare expenditure, without providing them clinical benefits.

The identification of patients for whom hospitalization might be dangerous would allow setting up alternative 


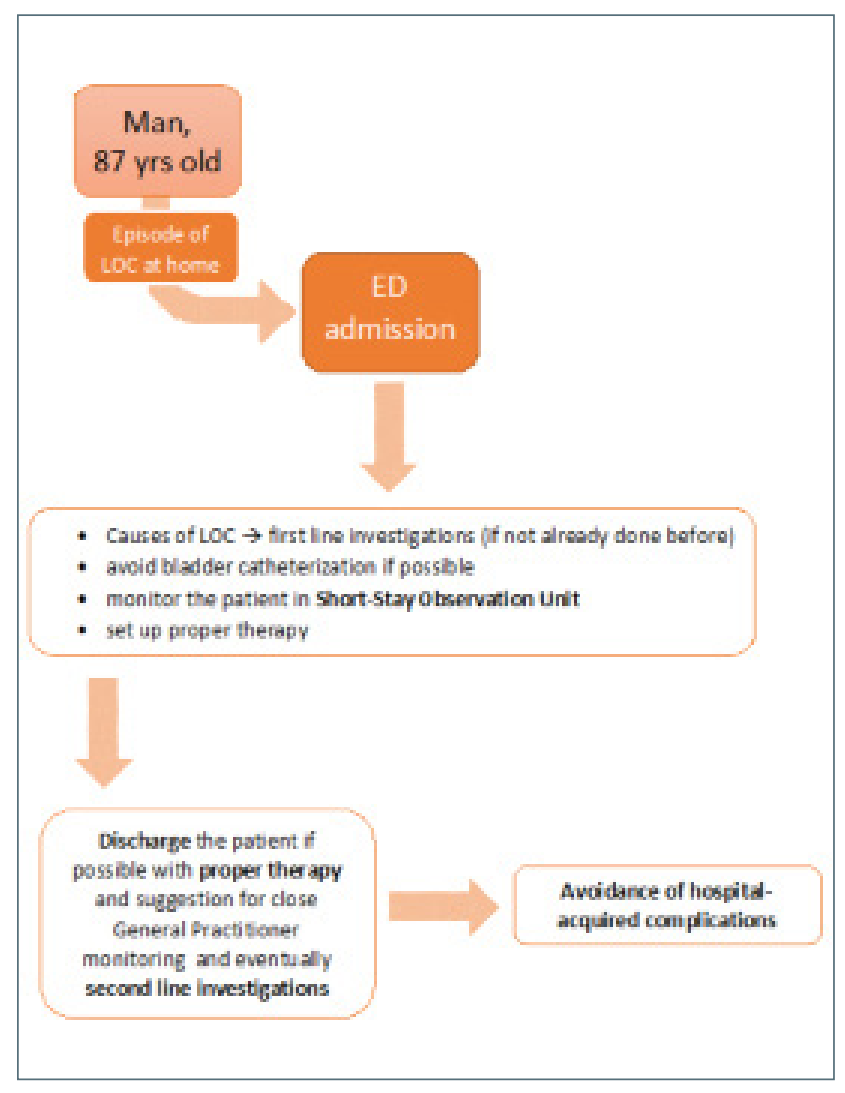

Figure 2. Ideal management of the patient. treatment strategies to answer to the unmet needs of frail people (Fig. 2). The expansion of home care programs and services could help in reducing inappropriate hospitalizations. Moreover, the novel models of care should include a prioritization and personalization of the interventions, with an appropriate balance of pros and cons based to individual needs.

\section{CONSENT FOR PUBLICATION}

Provided by the patient.

\section{References}

1 Cesari M, Calvani R, Marzetti E. Frailty in older persons. Clin Geriatr Med 2017;33:293-303. https://doi.org/10.1016/j. cger.2017.02.002

2 Cesari M, Prince M, Thiyagarajan JA, et al. Frailty: an emerging public health priority. J Am Med Dir Assoc 2016;17:18892. https://doi.org/10.1016/j.jamda.2015.12.016

3 Landi F, Onder G, Cesari M, et al. Comorbidity and social factors predicted hospitalization in frail elderly patients. J Clin Epidemiol 2004;57:832-6. https://doi.org/10.1016/j. jclinepi.2004.01.013

4 Maust DT, Kim HM, Chiang C, et al. Predicting risk of potentially preventable hospitalization in older adults with dementia. J Am Geriatr Soc 2019;67:2077-84. https://doi. org/10.1111/jgs. 16030 